Diagnostisches Arbeiten, das »durch und durch Erkennen« als Basis gezielter Interventionen, ist eine wichtige Aufgabe pädagogischer Arbeitsfelder. Dabei ergibt sich immer wieder auch die Problematik der Einordnung und Bewertung der erhobenen Informationen. Professionelles diagnostisches Arbeiten setzt daher fachliche Kompetenzen voraus, sich kritisch und reflektierend mit dem diagnostischen Handeln auseinanderzusetzen. Das in zweiter, aktualisierter und verbesserter Auflage erschienene »Lehrbuch diagnostischer Grundlagen der Heil- und Sonderpädagogik« von Christina Reichenbach und Helge Thiemann will diesen Kompetenzerwerb unterstützen.

Das Lehrbuch gliedert sich in drei Kernkapitel: Grundlagen, Methoden der Diagnostik und Praxisrelevante Anwendung und Professionalisierung. Im ersten Teil wird der diagnostische Prozess in seinen Teilschritten - Klärung der Fragestellung und Formulierung des Auftrags, Hypothesenbildung, Planung und Durchführung der diagnostischen Untersuchung, Analyse der Ergebnisse, Intervention und Evaluation / Bewertung - differenziert dargestellt. Der schrittweise Aufbau, die Praxisbeispiele und Reflexionsfragen leiten DiagnostikerInnen durch den Prozess und bieten mit Hilfe der Grafiken, Tabellen und Fotos eine fundierte Arbeitsgrundlage. Im zweiten Teil werden die Entstehung der Förderdiagnostik sowie der aktuelle Fachdiskurs aufgearbeitet. Die Darlegung des kritischen Diskurses regt die Auseinandersetzung mit der eigenen Rolle als Diagnostikerln in Bezug auf das Menschenbild, die zugrundeliegenden theoretischen Annahmen sowie die Interpretation der erhobenen Daten an.
Im Vordergrund des diagnostischen Prozesses steht die Aufgabe, den »Weg für das Erkennen zu finden«. Nach einer kurzen und verständlichen Einführung in statistische Grundlagen werden die Methoden-Test, schriftliche Befragung, Beobachtung, mündliche Befragung, Screening, diagnostisches Inventar, Arbeitsprodukt und Fehleranalyse - beschrieben und ausgewählte Verfahren für den heil- und sonderpädagogischen Arbeitsbereich vorgestellt.

Anhand von Praxisbeispielen wird die Erstellung von Gutachten und Förderplänen veranschaulicht. Die Autorlnnen setzen sich aber auch kritisch mit der Feststellung sonderpädagogischen Förderbedarfs auseinander und zeigen die dem föderalistischen System Deutschlands geschuldete Vielfalt an Regelungen und Rahmenbedingungen des Feststellungsprozesses auf. Sie verdeutlichen in dem Lehrbuch ihre positive Haltung zur prozessorientierten Diagnostik gegenüber einer selektiven »Platzierungsdiagnostik» und diskutieren in einem weiteren Kapitel die Möglichkeiten und Grenzen der Intelligenz- und Entwicklungsdiagnostik. Ihr Appell einer kritischen Auseinandersetzung mit Diagnostik mündet in den Überlegungen zur Qualifikation und Qualifizierung diagnostisch Tätiger. Die Übersicht kann die PraktikerInnen anregen, die eigenen Kompetenzen zu reflektieren und bietet aber zugleich Lehrenden in diesem Bereich eine Orientierung erforderlicher Kompetenzvermittlung. Die verantwortungsvolle Tätigkeit des Diagnostizierens zeigt sich auch in der Auseinandersetzung mit den ethischen Fragen, die dem Handeln zugrunde liegen sollte. Abschließend gehen die Autorlnnen der Frage nach, inwieweit Diagnostik und

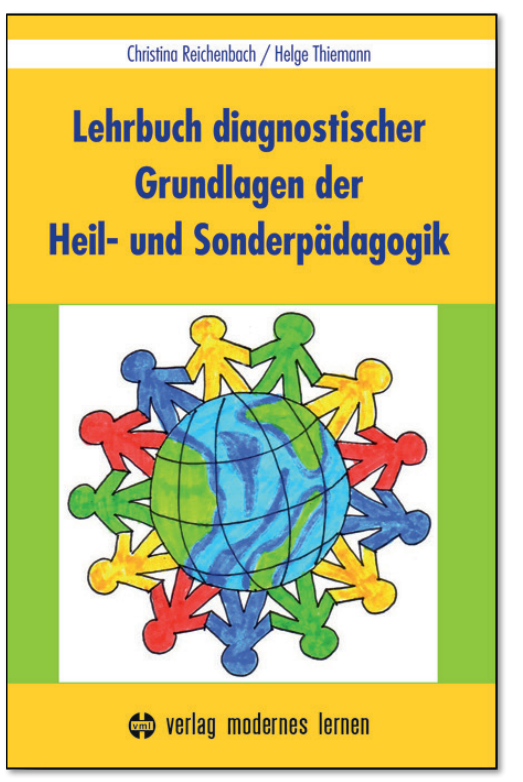

Inklusion im Widerspruch stehen. Sie konstatieren, dass eine prozessorientierte Diagnostik, welche Ansatzpunkte für eine gezielte Intervention und Unterstützung der Zielgruppe liefert, nicht im Widerspruch zum inklusiven Gedanken steht, sondern erst differenziertes pädagogisches Arbeiten ermöglicht.

Das Lehrbuch bietet mit den umfangreichen fachlichen Informationen und praktischen Beispielen eine fundierte Arbeitsgrundlage für die diagnostische Praxis wie für die Lehre. Die erforderliche Reflexion des eigenen Handelns wird durchgängig durch Leitfragen angeregt. Das Buch erfüllt das intendierte Ziel, handlungspraktische Kompetenzen zu vermitteln, da alle Kompetenzebenen - das Wissen, die Fertigkeiten, die Sozial- und die Selbstkompetenz konsequent angesprochen werden.

\section{Astrid Krus}

DOI 10.2378/ mot2020.artogd 